OPEN ACCESS

Edited by:

Livio Casarini,

University of Modena and Reggio

Emilia, Italy

Reviewed by:

Daniele Santi,

University of Modena and Reggio

Emilia, Italy

Danilo Cimadomo,

GENERA reparto di PMA della Clinica

Valle Giulia, Italy

*Correspondence:

Dangxia Zhou

zdxtougao@163.com

Juanzi Shi

shijuanzi123@126.com

Specialty section: This article was submitted to

Reproduction,

a section of the journa

Frontiers in Endocrinology

Received: 12 December 2019 Accepted: 04 March 2020

Published: 09 April 2020

Citation:

Xue X, Shi W, Zhou H, Tian L, Zhao Z, Zhou D and Shi J (2020) Cumulative Live Birth Rates According to Maternal Body Mass Index After First Ovarian Stimulation for in vitro Fertilization: $A$ Single Center Analysis of 14,782

Patients. Front. Endocrinol. 11:149. doi: 10.3389/fendo.2020.00149
Cumulative Live Birth Rates According to Maternal Body Mass Index After First Ovarian Stimulation for in vitro Fertilization: A Single Center Analysis of 14,782 Patients

\author{
Xia Xue ${ }^{1,2}$, Wenhao Shi ${ }^{2}$, Hanying Zhou ${ }^{2}$, Li Tian ${ }^{2}$, Zhenghao Zhao ${ }^{2}$, Dangxia Zhou ${ }^{1 *}$ and \\ Juanzi Shi ${ }^{2 *}$
}

${ }^{1}$ Department of Pathology, Medical School, Xi'an Jiaotong University, Xi'an, China, ${ }^{2}$ The Assisted Reproduction Center, Northwest Women's and Children's Hospital, Affiliated With Xi'an Jiaotong University, Xi'an, China

Objective: To investigate the cumulative live birth rates (CLBR) according to body mass index (BMI) in women undergoing their first in vitro fertilization (IVF).

Design: Retrospective cohort analysis.

Setting: An IVF clinic in a public hospital.

Patients: This is a retrospective study of 14,782 patients undergoing their first fresh IVF cycles and subsequent frozen embryo transfers in our clinic from January 2014 to January 2017. The follow-up for CLBR continued until January 2019. Patients with a $\mathrm{BMl}<18.5 \mathrm{~kg} / \mathrm{m}^{2}$ were considered to be underweight and those with a BMI $>24 \mathrm{~kg} / \mathrm{m}^{2}$ were considered to be overweight. Patients with a BMl $\geq 28 \mathrm{~kg} / \mathrm{m}^{2}$ were considered to be obese.

\section{Intervention(s): None.}

Primary Outcome Measure: The primary outcome was cumulative live birth rate (CLBR).

Result(s): This study illustrated the "inverted U shape" associations between body weight and IVF outcome (CLBR). The turning points in threshold analysis, as found by an automatic search, were BMls of 18.5 and $30.4 \mathrm{~kg} / \mathrm{m}^{2}$. The main finding of this retrospective data analysis is that the CLBR increased in underweight women, plateaued for normal weight and overweight women with a BMl between 18.5 and $30.4 \mathrm{~kg} / \mathrm{m}^{2}$, and decreased in obese women.

Conclusion(s): The data suggested an "inverted $U$ shape" association between BMl and CLBR. The CLBR increases in underweight women, plateaus in normal weight and overweight women, and then decreases in obese women.

Keywords: BMI, cumulative live birth rate, in vitro fertilization, overweight, underweight 


\section{INTRODUCTION}

Numerous studies have shown that a body mass index (BMI) that is either too high or too low is associated with a reduced probability of achieving pregnancy in women undergoing assisted reproductive technology (ART) (1). However, most of these studies are limited by being cycle-based (2-5), in which they only studied fresh cycles and reported the outcome in terms of live births per fresh cycle or embryo transfer. There cannot be a complete measure of an in vitro fertilization (IVF) treatment's success without a comprehensive analysis of the frozen embryo transfer (FET). In addition to affecting other aspects of the body's functions (6), BMI has an impact on the female reproductive system, both pre- and post-pregnancy. In this respect, a patient-based cumulative live birth rate (CLBR) that includes all fresh and frozen embryo transfers is a more suitable and more comprehensive measurement when reporting outcomes of ART (7). Before now, there were few reports using CLBR as the primary outcome measurement for the impact of BMI on IVF.

Current studies have adopted WHO classification criteria for BMI classification. The cutoffs for underweight $\left(<18.5 \mathrm{~kg} / \mathrm{m}^{2}\right)$, overweight (BMI $25-30 \mathrm{~kg} / \mathrm{m}^{2}$ ), and obese (BMI $>30 \mathrm{~kg} / \mathrm{m}^{2}$ ) were adopted to define a different population (8). However, we are not sure whether the threshold of BMI impact on CLBR is consistent with this criterion. Continuous variable data of BMI impact on CLBR is still not illustrated. There is still a lack of effective information, especially regarding patients who fall outside the normal BMI range in weight counseling before IVF.

Taking into account all the above-mentioned details, we set out to examine the relationship between BMI and first-time CLBR after a single stimulation among women undergoing IVF. Furthermore, we would like to figure out the turning point of the BMI's impact on CLBR, which can provide a valuable counseling resource for infertile women with abnormal BMIs.

\section{MATERIALS AND METHODS}

This is a retrospective study of 14,782 patients undergoing first fresh IVF/intracytoplasmic sperm injection (ICSI) cycles with subsequent frozen embryo transfer (FET) in our clinic from January 2014 to January 2017. The followup for CLBR continued for two years until January 2019. The study was approved by the Ethics Committee for the Clinical Application of Human Assisted Reproductive Technology of Northwest Women's and Children's Hospital (No. 2018002).

\section{Patients' Inclusion Criteria}

All first fresh IVF/ICSI patients $(n=14,782)$ who underwent subsequent FET from the same fresh oocyte retrieval in our clinic between January 2014 and January 2017 were included in the analysis.

The following cycles were excluded: (1) donated oocyte cycles, $n=14$; (2) pre-implantation genetic testing (PGT) cycles, $n=96$; (3) cycles that did not achieve live birth, with frozen embryos still in retrieval during this period, $n=292$; (4) cycles lost to followup, $n=30$; (5) cycles with induced abortion, $n=15$; (6) invalid BMI value, $n=132$.

The patients' BMIs were calculated as reported weight in kilograms per meter squared (reported height) at time of IVF start (before stimulation). The criteria for BMI categories were consistent with the international classifications of the World Health Organization (WHO), and adjusted according to the characteristics of an Asian population, which in general has a lower BMI than observed in non-Asian populations, with the BMI distribution shifted to the left (8). Thus, the patients with a $\mathrm{BMI}<18.5 \mathrm{~kg} / \mathrm{m}^{2}$ were considered to be underweight, those with a BMI $>24 \mathrm{~kg} / \mathrm{m}^{2}$ were considered to be overweight, and those with a BMI $\geq 28 \mathrm{~kg} / \mathrm{m}^{2}$ were considered to be obese. Finally, 14,215 patients were studied for subsequent analysis. Flowchart and data processing are displayed in Figure 1. Demographics and basal characteristics of patients are presented in Table $\mathbf{1}$.

\section{IVF Treatment}

The protocol for ovarian stimulation (OS) was determined individually according to the patient's age, BMI, basal folliclestimulating hormone, and antral follicle count (AFC). Most patients were treated with recombinant and/or urinary gonadotrophins (Gonal-F/PUREGON/Urofollitropin) in a long Gonadotropin-releasing hormone (GnRH) agonist or a GnRH antagonist protocol followed by IVF or ICSI. For women with diminished ovarian reserves, the mild ovulation protocol or luteal phase ovarian stimulation was attempted. Human menopausal gonadotrophin (HMG, Li Zhu, China) was added according to the patients' response to stimulation. Four thousands to ten thousands units of human chorionic gonadotrophin (hCG), or $250 \mu \mathrm{g}$ r-hCG (Merck Seronoy S.p.A.), were administered when two to three follicles were $>17 \mathrm{~mm}$. Oocyte retrieval was performed $36 \mathrm{~h}$ later by transvaginal ultrasonography-guided aspiration. The ovarian stimulation parameters for each group are displayed in Table $\mathbf{1 .}$

The procedures for oocyte extraction, embryo culture and the embryo scoring system were described in our previous studies $(9,10)$. Grades $1-3$ were considered useable embryos on day 3, and good-quality embryos were Grades 1-2. All IVF/intracytoplasmic sperm injection (ICSI) embryo transfer (ET) procedures were performed according to the following principles: embryo transfers were carried out on days 3 or 5 in fresh cycles; in cases with sufficient high-quality embryos (more than 3-4) on day 3, blastocyst transfer on day 5 was practiced; after fresh embryo transfer, patients' surplus embryos were vitrified; in cases of unsuccessful implantation during the fresh cycle, FET was carried out using surplus embryos; for luteal phase support, progesterone injection $(60 \mathrm{mg} /$ day $)$ was started after oocyte retrieval and maintained until a negative serum beta-hCG or the eighth week of pregnancy.

\section{Primary Outcome Measurement}

The primary outcome was CLBR, which was defined as at least one live birth resulting from one aspirated ART cycle in the fresh transfer or subsequent FET in relation to the number of 


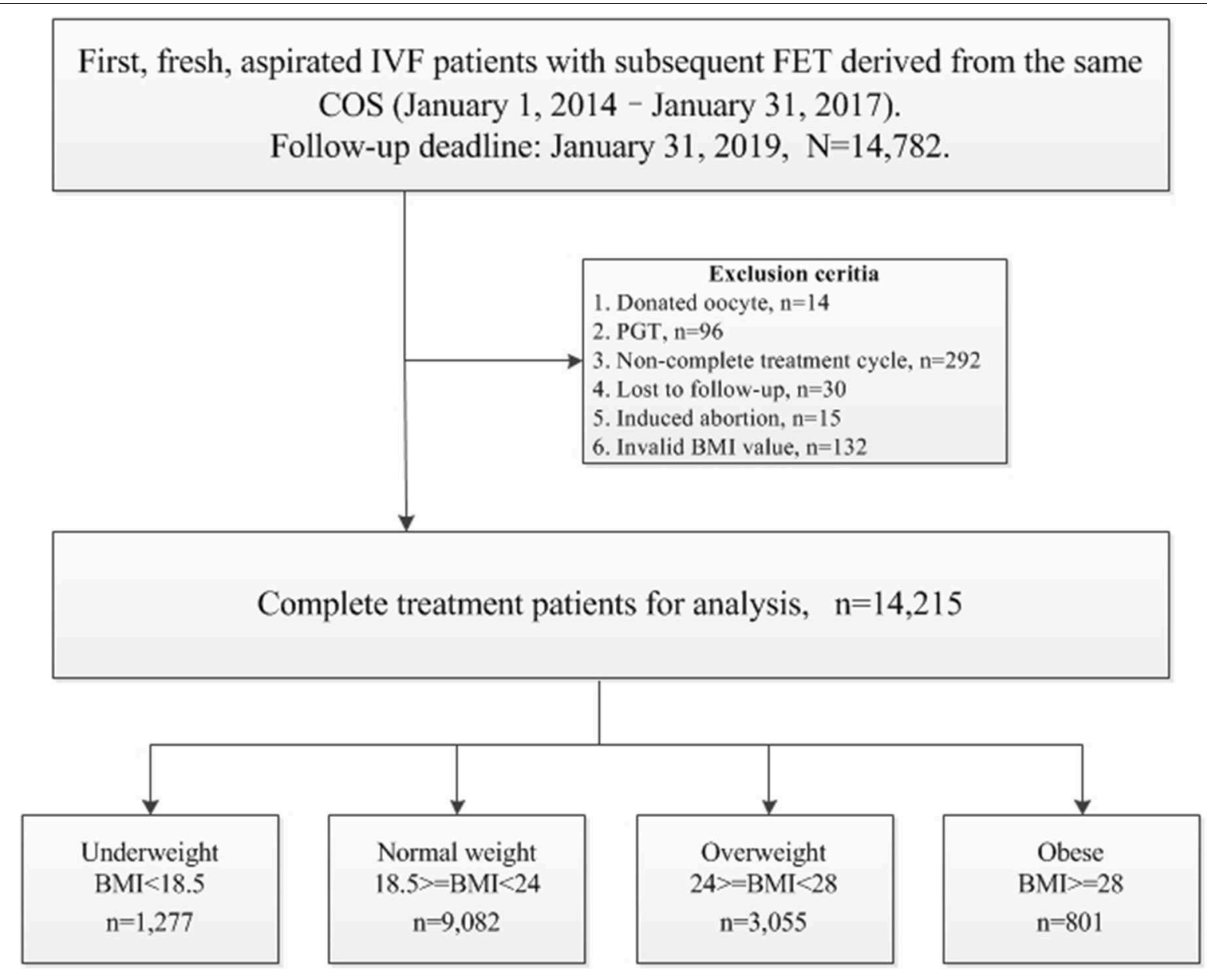

FIGURE 1 | Flow chart and data processing (BMI, $\left.\mathrm{kg} / \mathrm{m}^{2}\right)$.

oocytes retrieved. CLBR was evaluated by adding the pregnancies and live births achieved in the FETs to the ones obtained in the fresh cycle. Only the first delivery was considered in the analysis. One treatment cycle is defined as an oocyte retrieval and all transfers, fresh and frozen/thawed, derived from that ovarian stimulation. One complete treatment cycle refers to a treatment cycle that achieved live birth or a treatment cycle that transferred all embryos but failed to achieve live birth.

\section{Statistical Analysis}

The data processing and statistical analysis were conducted by EmpowerStats software (www.empowerstats.com, version R.3.4.3) and statistical software packages R. Multivariate. Logistic regression analyses were performed to evaluate the association between BMI and CLBR, with adjustments made for important co-variables and potential confounding factors (treatment year, patient's age, AFC, basal FSH, smoking, type of infertility, length of infertility, gravidity, parity, main etiology, stimulation protocol, total $\mathrm{Gn}$ dose, and starting FSH dose). A generalized additive model, where the outcome was the CLBR and the explanatory variable was a continuous BMI, was used to check for trend. A threshold analysis of BMI associated with CLBR was also performed.

\section{RESULTS}

Demographics and basal characteristics of IVF patients vary in Table 1. Obviously, there were significant differences among infertility-related parameters and anthropometrical variables by dividing patients according to BMI. Young women ( $<35$ years) were more likely to be underweight, have $<=2$ years of infertility, have tubal factor infertility, use the GnRH antagonist protocol, receive a lower dose of $\mathrm{Gn}$, and so on. These imbalanced variables may act as confounding factors and were adjusted in subsequent analysis. Statistically speaking, the CLBR significantly decreased among underweight (66.41\%), normal weight (65.79\%), overweight $(61.08 \%)$, and obese $(56.30 \%)$, as well as the number of day 3 embryos and the number of oocytes retrievals (Table 2). The oocyte output rate (oocytes retrieved/antral follicle count) also statistically significantly decreased among underweight 
TABLE 1 | Demographics and basal characteristics.

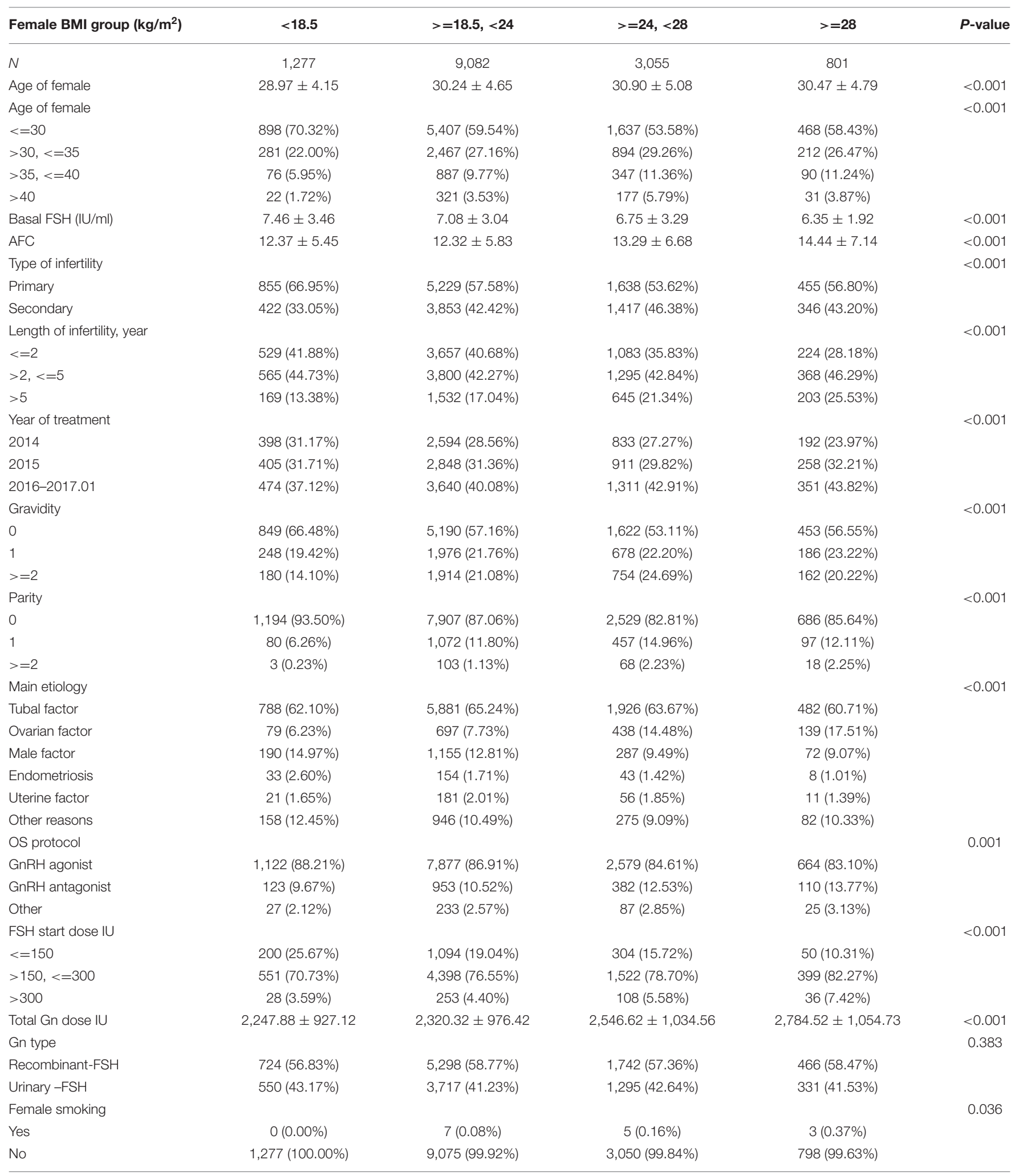

Mean + SD/N (\%).

AFC, antral follicle count; BMI, body mass index; FSH, follicle stimulating hormone; Gn, Gonadotropin; GnRH, Gonadotropin-releasing hormone; OS, ovarian stimulation. Created by EmpowerStats (www.empowerstats.com) and $R$. 
TABLE 2 | Oocytes and embryo parameters and cumulative live birth rates.

\begin{tabular}{|c|c|c|c|c|c|}
\hline Female BMI group $\left(\mathbf{k g} / \mathrm{m}^{2}\right)$ & $<18.5$ & $>=18.5,<24$ & $>=24,<28$ & $>=28$ & $P$-value \\
\hline$N$ & 1,277 & 9,082 & 3,055 & 801 & \\
\hline No. of oocytes & $11.99 \pm 6.96$ & $11.80 \pm 6.91$ & $11.45 \pm 7.05$ & $10.93 \pm 6.75$ & $<0.001$ \\
\hline No. of cleavage & $9.31 \pm 5.79$ & $9.16 \pm 5.84$ & $8.80 \pm 5.89$ & $8.52 \pm 5.87$ & $<0.001$ \\
\hline No. 2PN & $7.40 \pm 4.76$ & $7.21 \pm 4.69$ & $6.87 \pm 4.69$ & $6.55 \pm 4.48$ & $<0.001$ \\
\hline No. of day 3 useable embryos & $6.16 \pm 4.27$ & $6.01 \pm 4.23$ & $5.70 \pm 4.29$ & $5.42 \pm 4.15$ & $<0.001$ \\
\hline No. of day 3 good quality embryos & $3.67 \pm 3.33$ & $3.66 \pm 3.31$ & $3.50 \pm 3.35$ & $3.25 \pm 3.18$ & 0.001 \\
\hline No. of oocytes/AFC & $96.40 \%$ & $95.63 \%$ & $85.89 \%$ & $72.99 \%$ & $<0.001$ \\
\hline Cumulative live birth (rate \%) & $848(66.41 \%)$ & 5,975 (65.79\%) & $1,866(61.08 \%)$ & 451 (56.30\%) & $<0.001$ \\
\hline
\end{tabular}

AFC, antral follicle count; PN, pronucleus.

Mean + SD/N (\%), Created by EmpowerStats (www.empowerstats.com) and R.

TABLE 3 | Logistic regression analysis for cumulative live birth rates in BMl groups.

\begin{tabular}{lccc}
\hline & $\begin{array}{c}\text { Non-adjusted } \\
\text { OR }(\mathbf{9 5 \%} \mathbf{~ C l}) \\
\boldsymbol{P} \text {-value }\end{array}$ & $\begin{array}{c}\text { Adjust I } \\
\text { OR }(\mathbf{9 5 \%} \mathbf{C l}) \\
\boldsymbol{P} \text {-value }\end{array}$ & $\begin{array}{c}\text { Adjust II OR } \\
(\mathbf{9 5 \%} \mathbf{C l}) \boldsymbol{P} \text {-value }\end{array}$ \\
\hline $\begin{array}{l}\text { Female BMl group } \\
\left(\mathrm{kg} / \mathrm{m}^{2}\right)\end{array}$ & & & \\
$>=18.5,<24$ & $1(\mathrm{ref})$ & $1(\mathrm{ref})$ & $1(\mathrm{ref})$ \\
$<18.5$ & $1.03(0.91,1.16)$ & $0.92(0.80,1.04)$ & $0.86(0.73,1.02)$ \\
& 0.6637 & 0.1902 & 0.0888 \\
$>=24,<28$ & $0.82(0.75,0.89)$ & $0.82(0.74,0.89)$ & $0.86(0.76,0.97)$ \\
$>=28$ & $<0.0001$ & $<0.0001$ & 0.0118 \\
& $0.67(0.58,0.78)$ & $0.60(0.51,0.70)$ & $0.63(0.51,0.77)$ \\
& $<0.0001$ & $<0.0001$ & $<0.0001$ \\
\hline
\end{tabular}

OR (95\% Cl) P-value.

Adjusted I for female age, AFC, main etiology, OS protocol.

Adjusted II for year of treatment, patient's age, AFC, basal FSH, smoking, type of infertility, length of infertility, gravidity, parity, main etiology, OS protocol, Gn total dose, and FSH start dose.

Created by EmpowerStats (www.empowerstats.com) and R.

(96.40\%), normal weight (95.63\%), overweight $(85.89 \%)$, and obese (72.99\%).

A multiple variables regression analysis was performed, taking into account the fact that variables may act as confounding factors to the data described in Table 1. The unadjusted and adjusted odds ratios (OR) of CLBR with a 95\% confidence interval (CI) in different models are shown in Table 3. It was found that the BMI of underweight patients had no statistically significant effect on the CLBR (OR 0.92, 95\% CI 0.80-1.04, $p=$ 0.1902 ) compared with the normal weight group when adjusted for female age, AFC, main etiology, and OS protocol. The CLBR in overweight patients decreased significantly (OR 0.82, 95\% CI 0.74-0.89, $p<0.0001)$ compared with normal weight patients when adjusted for female age, AFC, main etiology, and OS protocol. However, the CLBR in the obese patients decreased by about $40 \%$ (OR $0.60,95 \%$ CI $0.51-0.70, p<0.0001$ ) when compared with the normal weight group in model 1 .

A generalized additive model (Figure 2) was built where the outcome was the CLBR and the explanatory variable was BMI (red dots). The blue dotted lines are the 95\% confidence intervals. The right model was adjusted for treatment year, patient's age, AFC, basal FSH, smoking habits, type of infertility, length of infertility, gravidity, parity, main etiology, OS protocol, Gn total dose, Gn type, and starting FSH dose. The CLBR increased linearly from a BMI of $15 \mathrm{~kg} / \mathrm{m}^{2}$ to a BMI of $\sim 18 \mathrm{~kg} / \mathrm{m}^{2}$, then showed a slowly declining segment. When BMI exceeded $\sim 30 \mathrm{~kg} / \mathrm{m}^{2}$, the CLBR fell sharply until the nadir. The association between CLBR and BMI looks like an inverted U-shape. The turning points (BMI 18.5 and $30.4 \mathrm{~kg} / \mathrm{m}^{2}$ ) were determined using threshold analysis (Table 4).

The oocyte retrieval (number of oocytes/AFC) and embryo development (number of $2 \mathrm{pn}$, day 3 embryos and good embryos/number of oocytes) in different subgroups of BMI were displayed in Figure 3. The differences between BMI subgroups were mainly expressed in oocyte retrieval, rather than embryo development indicators, such as 2 pns, day 3 embryos, and good day 3 embryos per oocyte. The subgroup analysis was performed according to the ranges of AFC, patient's age, and stimulation protocol in Table 5, in which the CLBR showed a trend similar to the general trend in all subgroups with the addition of women over 35. The multivariable fractional polynomials analysis was conducted in Table S1, which could be helpful to highlight which parameters better predict the CLBR.

\section{DISCUSSION}

The main finding of this retrospective data analysis is that the CLBR increased among underweight women, plateaued in normal weight and overweight women, and then decreased in obese women. The highest CLBR was observed among women with a BMI of $18.5 \mathrm{~kg} / \mathrm{m}^{2}$, surrounded by normal weight and underweight women. Another finding was that oocyte output rate decreased as $\mathrm{BMI}$ increased. However, there is no difference in the embryo development indicators.

In 2004, Fedorcsák et al. (11) first reported the CLBR of underweight and overweight women in a large cohort of women who underwent IVF, which is consistent with the present study. Rather than calculating 

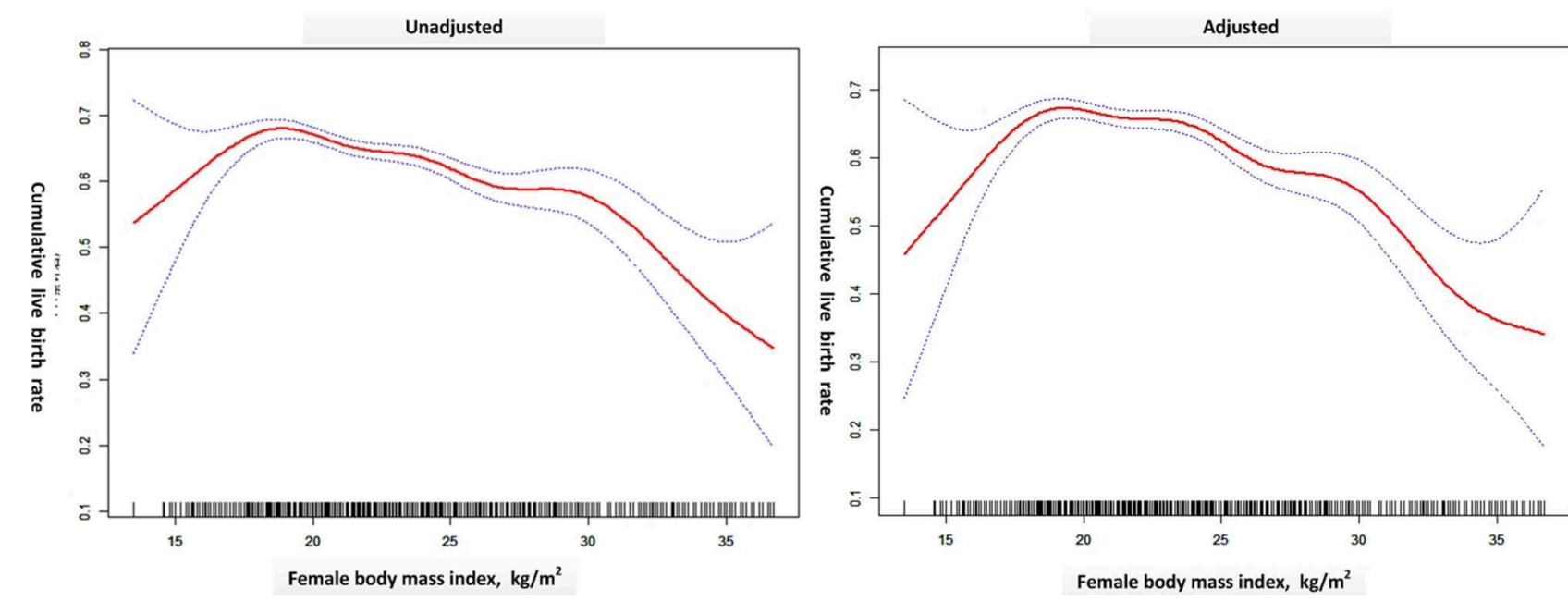

FIGURE 2 | A generalized additive model was built where the outcome was the CLBR and the explanatory variable was BMI (red dots). The blue dotted lines are the 95\% confidence intervals. The right model was adjusted for treatment year, patient's age, AFC, basal FSH, smoking habits, type of infertility, length of infertility, gravidity, parity, main etiology, OS protocol, Gn total dose, Gn type, and FSH start dose. Created by EmpowerStats (www.empowerstats.com) and R.

TABLE 4 | Threshold analysis of BMl associated with cumulative live birth rate.

\begin{tabular}{lc}
\hline Outcome & CLBR \\
\hline $\begin{array}{l}\text { Model I } \\
\text { One-line slope }\end{array}$ \\
Model II \\
$\quad 0.96(0.95,0.97)<0.0001$ \\
$\quad$ Turing points (K1,K2) \\
$\quad$ K 1 Slope 1 \\
K1-K2 Slope 2 & $18.5,30.4$ \\
$>$ K2 Slope 3 & $1.26(1.12,1.41)<0.0001$ \\
Slope 1-Slope 2 & $0.96(0.94,0.97)<0.0001$ \\
Slope 3-Slope 2 & $0.88(0.74,1.05) 0.1589$ \\
LRT test & $1.31(1.16,1.48)<0.0001$ \\
\hline
\end{tabular}

Results in table: OR (95\% Cl) P-value. Adjusted for female age, AFC, main etiology, OS protocol.

Created by EmpowerStats (www.empowerstats.com) and $R$.

CLBR with the Kaplan-Meier method after three cycles of IVF like Fedorcsak, our calculation was a patientbased outcome measurement stressing the first fresh aspirated IVF treatment, including all fresh and frozen embryo transfers. Moreover, the spline smoothing plot of CLBR according to BMI was first presented to illustrate their relationship.

The "inverted U shape" associations between BMI and IVF outcome have been shown in several studies $(2,4,12,13)$. While obesity has been demonstrated to impair pregnancy outcome, more issues focus on underweight and overweight women $\left(24-28 \mathrm{~kg} / \mathrm{m}^{2}\right)$. Our results suggested that being underweight had no significant effect on CLBR and being overweight had a slight impact and decreased CLBR, which is consistent with the results from the 2008-2013 Society for Assisted Reproductive Technology registry in the United States $(2,4)$.
Unfortunately, in some studies (14-16), the obese group was included in the overweight group for analysis rather than the subdivision analysis. Though the mechanism of the negative effect of BMI on IVF outcome is unclear, the relationship may have a threshold effect on the CLBR by accumulative BMI. Our study figured out the cut-off value of $30.4 \mathrm{~kg} / \mathrm{m}^{2}$, which means the IVF outcome of women with a BMI of more than $30.4 \mathrm{~kg} / \mathrm{m}^{2}$ would be severely impaired by excessive BMI. At the other body weight extreme, women with a BMI of less than $18.5 \mathrm{~kg} / \mathrm{m}^{2}$ would have impaired CLBR due to low BMI.

Most studies (4, 17-19) failed to demonstrate the association between low BMI and impaired IVF outcome. However, Cai et al. (3) recently concluded that low BMI is associated with negative outcomes in fresh transfer cycles, especially in women of advanced age. Obviously, in Cai's study only fresh transfer cycles were included, and frozen embryo transfers and cases with canceled fresh transfers were both excluded. Biases of population selection may cause inconsistency with our results. In addition, the outcome measurement in Cai's study adopted the simple live birth rate rather than the CLBR used in our study. Contrary to the results of Cai's study in the same Chinese population, low BMI was not associated with reduced CLBR (OR 0.89, 95\% CI 0.75-1.06, $p=0.1891$ ). Instead, the highest CLBR was observed among women with a BMI of $18.5 \mathrm{~kg} / \mathrm{m}^{2}$.

Maternal BMI is significantly associated with CLBR in the patients with first IVF/ICSI treatment. The CLBR remains relatively constant in IVF patients in the normal and overweight range (BMI 18.5-30.4). Therefore, for these patients, it is not necessary to be concerned about the impact of BMI on CLBR. Being underweight may have a limited impact on IVF success (namely CLBR), however, and gaining weight still improves the chances of CLBR (about $26 \%$ per unit of BMI). Being obese has a significant impact on IVF success, with a CLBR loss of about $12 \%$ 

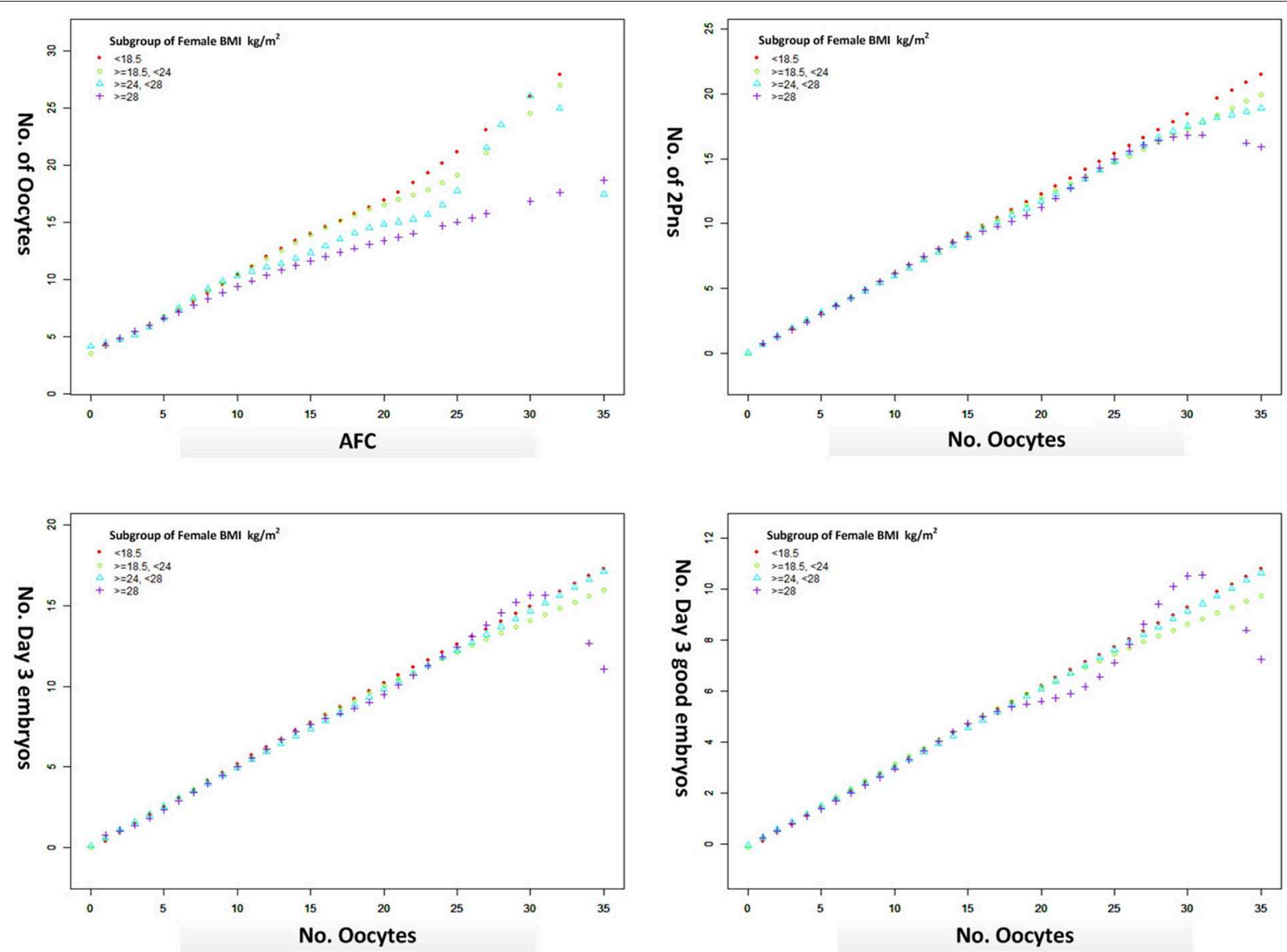

FIGURE 3 | The oocyte retrieval (number of oocytes/AFC) and embryo development (number of 2pn, day 3 embryos and good embryos/no. oocytes) in different subgroups of BMl from a generalized additive model, adjusted for female age, main etiology, and OS protocol.

TABLE 5 | Subgroup analysis of CLBR according to AFC, maternal age, and stimulation protocol.

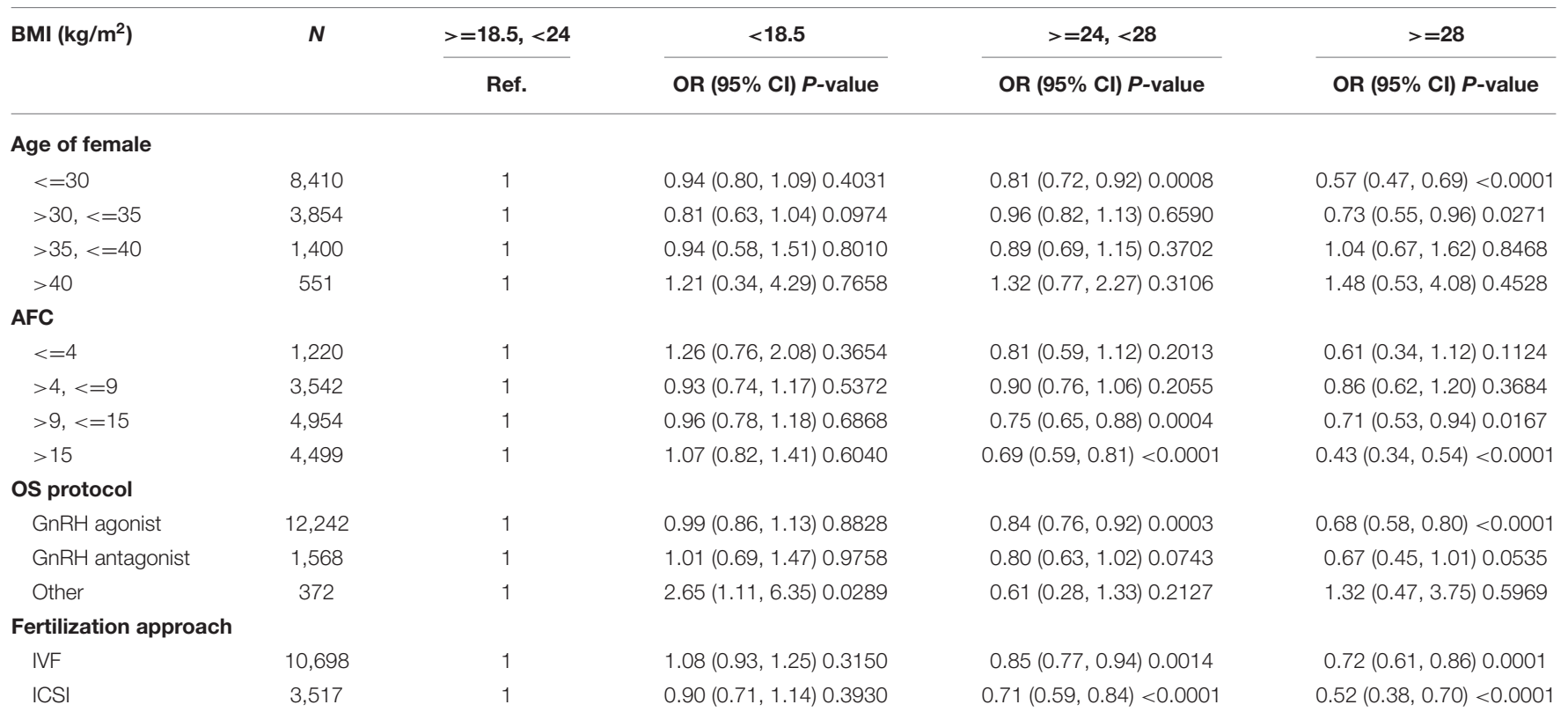


for every one unit increase of BMI. This provides specific and effective data in support of weight counseling for infertile women before IVF treatment.

Our data suggested that the oocyte output rate (number of oocytes/AFC) was damaged due to the excessive BMI. However, there was no difference in the embryo development indicators. Consistent with the literature, obesity was associated with ovulatory dysfunction and higher doses of ovarian stimulation in IVF $(20,21)$, leading to a lower chance of oocyte retrieval. Although the evidence from both clinical data and animal studies suggests obesity negatively impacts the developmental competence of oocytes $(22,23)$, there are still questions about the pathophysiology underlying these findings because not all studies took into account the damage in embryo quality $(24,25)$.

Limitations are related to the retrospective nature of the study, and analysis from a single center also weakens the universality of our observations. The results of the study should also be interpreted with caution when considering other populations and areas because Asian populations in general have a lower BMI than observed in non-Asian populations, with a BMI distribution shifted to the left (8). The variance of BMI during IVF treatment and pregnancy, which was not involved in the study, may also be a potential confounding impact on the live birth.

\section{CONCLUSION}

In conclusion, there is an "inverted $U$ shape" association between body weight and IVF outcome (CLBR). CLBR increased among underweight women, plateaued in normal weight and overweight women with a BMI of 18.5-30.4, and then decreased in obese women proportionally to the accumulation of BMI. The present study may therefore be helpful for IVF patients with abnormal BMI to predict pregnancy outcome (CLBR) when attending weight counseling before initiating IVF cycles.

\section{REFERENCES}

1. Sarais V, Pagliardini L, Rebonato G, Papaleo E, Candiani M, Vigano P. A comprehensive analysis of body mass index effect on in vitro fertilization outcomes. Nutrients. (2016) 8:109. doi: 10.3390/nu8030109

2. Provost MP, Acharya KS, Acharya CR, Yeh JS, Steward RG, Eaton JL, et al. Pregnancy outcomes decline with increasing body mass index: analysis of 239,127 fresh autologous in vitro fertilization cycles from the 2008-2010 Society for Assisted Reproductive Technology registry. Fertil Steril. (2016) 105:663-9. doi: 10.1016/j.fertnstert.2015.11.008

3. Cai J, Liu L, Zhang J, Qiu H, Jiang X, Li P, et al. Low body mass index compromises live birth rate in fresh transfer in vitro fertilization cycles: a retrospective study in a Chinese population. Fertil Steril. (2017) 107:422-9 e2. doi: 10.1016/j.fertnstert.2016.10.029

4. Kawwass JF, Kulkarni AD, Hipp HS, Crawford S, Kissin DM, Jamieson DJ. Extremities of body mass index and their association with pregnancy outcomes in women undergoing in vitro fertilization in the United States. Fertil Steril. (2016) 106:1742-50. doi: 10.1016/j.fertnstert.2016.08.028

\section{DATA AVAILABILITY STATEMENT}

All datasets generated for this study are included in the article/Supplementary Material.

\section{AUTHOR CONTRIBUTIONS}

$\mathrm{XX}$ : writing the article, study conception and design, and critical review of the article. WS, LT, ZZ, and HZ: data processing. DZ and JS: supervising the study. All authors approved the manuscript at submission format.

\section{FUNDING}

This study was funded by General Project of Science and Technology Department of Shaanxi Province - Social Development Field (No. 2018SF-260) and Special Research Fund of Clinical Medicine of Chinese Medical Association - Research and Development Project of Young Doctors in Reproductive Medicine (17020120681). This study was also funded by National Natural Science Funding of China (No. 81673224) and Natural Science Funding of Shaanxi Province (No. 2019JM029).

\section{ACKNOWLEDGMENTS}

We thank all the clinicians, scientists, and embryologists in the Northwest Women and Children's Hospital for their assistance with the data collection, and all the patients for their contribution to this study. We gratefully thank Dr. Xinglin Chen from Department of Epidemiology and Biostatistics, X\&Y Solutions, Inc. in Boston for her contribution to the statistical support.

\section{SUPPLEMENTARY MATERIAL}

The Supplementary Material for this article can be found online at: https://www.frontiersin.org/articles/10.3389/fendo. 2020.00149/full\#supplementary-material

5. Friedler S, Cohen O, Liberty G, Saar-Ryss B, Meltzer S, Lazer T. Should high BMI be a reason for IVF treatment denial? Gynecol Endocrinol. (2017) 33:853-6. doi: 10.1080/09513590.2017.1327042

6. Imterat M, Agarwal A, Esteves SC, Meyer J, Harlev A. Impact of Body Mass Index on female fertility and ART outcomes. Panminerva Med. (2019) 61:58-67. doi: 10.23736/S0031-0808.18.03490-0

7. Maheshwari A, McLernon D, Bhattacharya S. Cumulative live birth rate: time for a consensus? Hum Reprod. (2015) 30:2703-7. doi: 10.1093/humrep/dev263

8. WHO Expert Consultation. Appropriate body-mass index for Asian populations and its implications for policy and intervention strategies. Lancet. (2004) 363:157-63. doi: 10.1016/S0140-6736(03)15268-3

9. Shi W, Zhang S, Zhao W, Xia X, Wang M, Wang H, et al. Factors related to clinical pregnancy after vitrified-warmed embryo transfer: a retrospective and multivariate logistic regression analysis of 2313 transfer cycles. Hum Reprod. (2013) 28:1768-75. doi: 10.1093/humrep/det094

10. Shi W, Xue X, Zhang S, Zhao W, Liu S, Zhou H, et al. Perinatal and neonatal outcomes of 494 babies delivered from 972 vitrified embryo transfers. Fertil Steril. (2012) 97:1338-42. doi: 10.1016/j.fertnstert.2012.02.051 
11. Fedorcsak P, Dale PO, Storeng R, Ertzeid G, Bjercke S, Oldereid N, et al. Impact of overweight and underweight on assisted reproduction treatment. Hum Reprod. (2004) 19:2523-8. doi: 10.1093/humrep/deh485

12. Kasim K, Roshdy A. Body mass index and pregnancy outcome after assisted reproduction treatment. Int J Reprod Med. (2014) 2014:257974. doi: 10.1155/2014/257974

13. Veleva Z, Tiitinen A, Vilska S, Hyden-Granskog C, Tomas C, Martikainen $\mathrm{H}$, et al. High and low BMI increase the risk of miscarriage after IVF/ICSI and FET. Hum Reprod. (2008) 23:878-84. doi: 10.1093/humrep/ den017

14. Supramaniam PR, Mittal M, McVeigh E, Lim LN. The correlation between raised body mass index and assisted reproductive treatment outcomes: a systematic review and meta-analysis of the evidence. Reprod Health. (2018) 15:34. doi: 10.1186/s12978-018-0481-z

15. Caillon H, Freour T, Bach-Ngohou K, Colombel A, Denis MG, Barriere P, et al. Effects of female increased body mass index on in vitro fertilization cycles outcome. Obes Res Clin Pract. (2015) 9:382-8. doi: 10.1016/j.orcp.2015. 02.009

16. Purewal S, Chapman SCE, van den Akker OBA. A systematic review and meta-analysis of lifestyle and body mass index predictors of successful assisted reproductive technologies. J Psychosom Obstet Gynaecol. (2019) 40:218. doi: 10.1080/0167482X.2017.1403418

17. Lashen H, Ledger W, Bernal AL, Barlow D. Extremes of body mass do not adversely affect the outcome of superovulation and in-vitro fertilization. Hum Reprod. (1999) 14:712-5. doi: 10.1093/humrep/14.3.712

18. Wittemer C, Ohl J, Bailly M, Bettahar-Lebugle K, Nisand I. Does body mass index of infertile women have an impact on IVF procedure and outcome? J Assist Reprod Genet. (2000) 17:547-52. doi: 10.1023/A:1009402130903

19. Singh N, Gupta P, Mittal S, Malhotra N. Correlation of body mass index with outcome of in vitro fertilization in a developing country. Arch Gynecol Obstet. (2012) 285:259-63. doi: 10.1007/s00404-011-2013-8
20. Broughton DE, Moley KH. Obesity and female infertility: potential mediators of obesity's impact. Fertil Steril. (2017) 107:840-7. doi: 10.1016/j.fertnstert.2017.01.017

21. Practice Committee of the American Society for Reproductive Medicine. Obesity and reproduction: a committee opinion. Fertil Steril. (2015) 104:111626. doi: 10.1016/j.fertnstert.2015.08.018

22. Finger BJ, Harvey AJ, Green MP, Gardner DK. Combined parental obesity negatively impacts preimplantation mouse embryo development, kinetics, morphology and metabolism. Hum Reprod. (2015) 30:208496. doi: 10.1093/humrep/dev142

23. Purcell SH, Moley KH. The impact of obesity on egg quality. J Assist Reprod Genet. (2011) 28:517-24. doi: 10.1007/s10815-011-9592-y

24. Metwally M, Cutting R, Tipton A, Skull J, Ledger WL, Li TC. Effect of increased body mass index on oocyte and embryo quality in IVF patients. Reprod Biomed Online. (2007) 15:532-8. doi: 10.1016/S1472-6483(10)60385-9

25. Depalo R, Garruti G, Totaro I, Panzarino M, Vacca MP, Giorgino F, et al. Oocyte morphological abnormalities in overweight women undergoing in vitro fertilization cycles. Gynecol Endocrinol. (2011) 27:8804. doi: $10.3109 / 09513590.2011 .569600$

Conflict of Interest: The authors declare that the research was conducted in the absence of any commercial or financial relationships that could be construed as a potential conflict of interest.

Copyright @ 2020 Xue, Shi, Zhou, Tian, Zhao, Zhou and Shi. This is an open-access article distributed under the terms of the Creative Commons Attribution License (CC $B Y)$. The use, distribution or reproduction in other forums is permitted, provided the original author(s) and the copyright owner(s) are credited and that the original publication in this journal is cited, in accordance with accepted academic practice. No use, distribution or reproduction is permitted which does not comply with these terms. 